\title{
Diagnosis of the presence of lymph node metastasis and decision of operative indication using fluorodeoxyglu- cose - positron emission tomography and computed tomography in patients with primary lung cancer
}

\author{
Hiroaki Toba ${ }^{a}$, Kazuya Kondo ${ }^{a}$, Hideki Otsuka ${ }^{b}$, Hiromitsu Takizawa \\ Koichiro Kenzaki ${ }^{a}$, Shoji Sakiyama ${ }^{a}$, and Akira Tangoku ${ }^{a}$ \\ a Department of Thoracic and Endocrine Surgery and Oncology, and ${ }^{\mathrm{b}}$ Department of Radiology, \\ Institute of Health Biosciences, the University of Tokushima Graduate School, Tokushima, Japan
}

\begin{abstract}
Objectives : We evaluate whether integrated fluorodeoxyglucose-positron emission tomography and computed tomography (FDG-PET/CT) scan can diagnose the presence of lymph node metastasis more accurately than computed tomography (CT) scan alone. Methods : Forty-two patients with lung cancer preoperatively underwent integrated PET/CT scan using FDG and CT scan and underwent pulmonary resection and lymph node dissection. We judged cases as lymph node metastasis if the lymph node visually accumulated FDG at PET/CT scan and measured $1 \mathrm{~cm}$ or greater in the short axis at CT scan. We retrospectively analyzed whether our judgments in each modality were consistent with the pathological diagnosis. Results : Two-hundred and seventeen stations of lymph node were dissected and 21 stations $(9.7 \%)$ were histologically diagnosed as positive metastasis. Thirty-two stations of lymph node visually accumulated FDG at PET/CT scan and 17 stations measured $1 \mathrm{~cm}$ or greater in the short axis at $\mathrm{CT}$ scan. Concerning the diagnosis of lymph node metastasis, PET/CT scan showed significantly higher sensitivity than CT scan ( $81 \%$ vs. $48 \%, p=0.024)$. The false-positive rate was significantly high in PET-positive lymph nodes measuring less than $1 \mathrm{~cm}$ in diameter. There were 4 falsenegative lymph nodes with both scans. All of these were less than $7 \mathbf{~ m m}$ in diameter and had a low percentage of metastatic foci in the lymph node. Concerning the diagnosis of $\mathrm{N}$ staging, there was no significant difference between PET/CT scan and CT scan (83\% vs. 69\%, $p=0.124$ ). However, the identification of $\mathrm{N} 2$ disease at PET/CT scan was significantly more accurate than that at CT scan $(100 \%$ vs. $38 \%, p=0.031)$. Conclusions : PET/CT is superior to CT scan in lymph node staging. However, because the false-positive rate is high in PET-positive lymph nodes measuring less than $1 \mathrm{~cm}$ in diameter, we think that clinical background should be considered and other modalities or histological examinations should be undertaken if necessary. J. Med. Invest. 57 : 305-313, August, 2010
\end{abstract}

Keywords : fluorodeoxyglucose-positron emission tomography and computed tomography scan, computed tomography scan, lung cancer, lymph node metastasis, diagnosis

Received for publication April 21, 2010 ; accepted July 9, 2010.

Address correspondence and reprint requests to Kazuya Kondo, Department of Thoracic and Endocrine Surgery and Oncology, Institute of Health Biosciences, the University of Tokushima Graduate School, Kuramoto-cho 3-18-15, Tokushima 770-8503, Japan and Fax : +81-88-633-7144.

\section{INTRODUCTION}

Accurate diagnosis of lymph node metastasis is very important in deciding the optimal treatment 
of lung cancer. Recently, positron emission tomography and computed tomography (PET/CT) scan has been undertaken in many facilities and its efficacy for the diagnosis of accurate staging of lung cancer has been reported (1-2). Gould MK. et al. (3) reported that PET scan was significantly better than computed tomography (CT) scan in a meta-analysis. The PLUS multi-center randomized trial also proved the effectiveness of PET scan in preoperative patients with suspected lung cancer (4). Subsequently, Birm Ö et al. (5) reported the same results in a meta-analysis and, recently, it has been indicated that PET scan should be undertaken for patients with lung cancer with regard to mediastinal and extrathoracic staging, and it is recommended as grade IB by a standardized method 'Methodology for Lung Cancer Evidence Review and Guideline Development' in the ACCP guidelines (6).

Since PET/CT scan was introduced in our facility in December 2005, we have used it for lung cancer patients to diagnose preoperative staging, recurrence, and to judge the effectiveness of induction therapy. We retrospectively evaluated the effectiveness of PET/CT scan for preoperative lymph node staging for lung cancer patients compared with that of CT scan.

\section{MATERIALS AND METHODS}

\section{Patients}

Between December 2005 and April 2007, 42 consecutive patients with a histologic diagnosis of lung cancer, who underwent a curative operation at Tokushima University Hospital, were retrospectively reviewed. All patients preoperatively underwent both integrated PET/CT scan using fluorodeoxyglucose (FDG) and chest CT scan including chest X-ray and brain MRI as well as operative indication being assessed. The protocol of this study was not submitted to Institutional Review Board because FDG$\mathrm{PET} / \mathrm{CT}$ scan and chest CT scan were routine preoperative examination and this study was retrospective one. The patients underwent pulmonary resection and lymph node dissection, which was a radical operation. None of the patients in the study received preoperative chemotherapy or radiotherapy. The histologic type of lung cancer was adenocarcinoma in 30 patients, squamous cell carcinoma in 8 , large cell carcinoma in 1 , small cell carcinoma in 2 , and pleomorphic carcinoma in 1 . The pathologic $\mathrm{N}$ stages were $\mathrm{N} 0$ in $32, \mathrm{~N} 1$ in $2, \mathrm{~N} 2$ in 8 , and $\mathrm{N} 3$ in 0
(Table 1). The lymph nodes were classified according to the original lymph node map of lung cancer (7).

Table 1. Characteristics of the patients

\begin{tabular}{lc}
\hline Variable & Data \\
\hline NO. of patients & 42 \\
Age (yrs) & $68.0 \pm 9.5$ \\
Sex & \\
Male & 25 \\
Female & 17 \\
Tumor location & \\
Right upper lobe & 14 \\
Right middle lobe & 4 \\
Right lower lobe & 9 \\
Left upper lobe & 5 \\
Left lower lobe & 10 \\
Histology & \\
Adenocarcinoma & 30 \\
Squamous cell carcinoma & 8 \\
Large cell carcinoma & 1 \\
Small cell carcinoma & 2 \\
Pleomorphic carcinoma & 1 \\
Pathologic N stage & 32 \\
N0 & 2 \\
N1 & 8 \\
N2 & \\
N3 & \\
\hline
\end{tabular}

FDG-PET/CT scan protocol and image interpretation

The patients were required to fast for 6 hours and avoid strenuous work or exercise for 24 hours before PET/CT scan. Sixty minutes after the intravenous injection of FDG (3.7 MBq/ $\mathrm{kg}$ body weight), PET/CT scan was performed with an Aquiduo (Toshiba Medical Systems, Tokyo, Japan). Patients were imaged from the skull base to the mid-thigh level. The technical parameters used for the CT scan were a detector row configuration of $16 \times 1.25 \mathrm{~mm}$, a helical pitch of 15 , a gantry rotation speed of 0.5 $\mathrm{s}$, peak voltage of $120 \mathrm{kVp}$, a tube load of $50 \mathrm{~mA}$ and slice thickness of $2 \mathrm{~mm}$. An emission scan was acquired immediately following the CT scan for 2 min per bed position in 3D mode. The images were usually reconstructed using ordered subset expectation maximization selecting 4 iterations and 12 subsets, a $128 \times 128$ matrix, and post-smoothing with an $8 \mathrm{~mm}$ Gaussian filter. The reconstructed 
spatial resolution after smoothing was $9.2 \mathrm{~mm}$.

Abnormal FDG uptake was defined as greater than background activity in surrounding normal tissue excluding physiologic uptake sites. Regions of interest (ROI) were placed on visible uptake sites and standardized uptake values (SUV) were calculated as

SUV = decay-corrected activity $(\mathrm{kBq}) /$ tissue volume (ml)/injected-FDG activity (kBq)/body weight (g). In this study, the maximum SUV was used to minimize of partial volume effect and assure reproducibility of measurements.

\section{CT scan protocol and image interpretation}

Multidetector CT scan was performed with an Aquilion 16 (Toshiba Medical Systems, Tokyo, Japan). The technical parameters used for the CT scan were a detector row configuration of $16 \times 0.5$ $\mathrm{mm}$, a helical pitch of 15 , a gantry rotation speed of $0.5 \mathrm{~s}$, peak voltage of $120 \mathrm{kVp}$, and a tube load of 100-400 mA and slice thickness of $1 \mathrm{~mm}$. The window level and width were set at -500 Hounsefield units (HU), and $1500 \mathrm{HU}$.

\section{Criteria of lymph node metastasis}

We judged the lymph node station as metastasis if FDG was visually accumulated greater than background activity in surrounding normal tissue excluding physiologic uptake sites at PET/CT scan. At the same time, we measured the short-axis diameter of the largest lymph node at its station. We also judged the lymph node station as metastasis if the short-axis diameter of the lymph node was greater than $1 \mathrm{~cm}$ at axial view of CT scan as in previous reports $(8,9)$. Two chest surgeons $\mathrm{HT}$ and KK analyzed PET/CT scan by consensus while referring to reports made by several experienced nuclear medicine physicians. Two chest surgeons HT and $\mathrm{KK}$ also analyzed CT scan by consensus while referring to reports made by several radiologists. Discrepancies were resolved after consultation.

\section{Histological Examination}

The dissected lymph nodes were examined histologically using formalin-fixed and paraffin-embedded sections and the largest section in each lymph node was cut and examined with hematoxylin and eosin staining with a microscope.

\section{Statistical Analysis}

True-positive (TP), true-negative (TN), falsepositive $(\mathrm{FP})$, and false-negative $(\mathrm{FN})$ results of
PET/CT scan and CT scan for lymph node metastasis were compared with the results of pathologic diagnosis. Sensitivity was calculated as TP/TP+FN, specificity as $\mathrm{TN} / \mathrm{TN}+\mathrm{FP}$, positive predictive value (PPV) as TP/TP+FP, negative predictive value (NPV) as TN/TN+FN, and accuracy as TP+TN/ total. All data were analyzed for significance by the 2-tailed Student's $t$ test using the SPSS 11.0 for Windows software (SPSS Japan Inc., Tokyo, Japan).

\section{RESULTS}

In the 217 dissected lymph node stations, 21 stations $(9.7 \%)$ were histologically diagnosed as positive metastasis. Thirty-two lymph node stations visually accumulated FDG at PET/CT scan and the mean value of maximum SUV of those lymph node stations was between 1.7 and 8.6 (mean \pm SD, 3.7 \pm 1.6). PET/CT scan yielded TP in 17 lymph node stations, FP in 15, FN in 4, and TN in 181 (Table 2).

Table 2. PET/CT and CT analysis with pathologic diagnosis

\begin{tabular}{|c|c|c|c|}
\hline Variable & With metastasis & Without metastasis & Total \\
\hline \multicolumn{4}{|c|}{$\mathrm{PET} / \mathrm{CT}$ diagnosis } \\
\hline Positive & 17 true-positive & 15 false-positive & 32 \\
\hline Negative & 4 false-negative & 181 true-negative & 185 \\
\hline Total & 21 & 196 & 217 \\
\hline \multicolumn{4}{|c|}{ CT diagnosis } \\
\hline Positive & 10 true-positive & 7 false-positive & 17 \\
\hline Negative & 11 false-negative & 189 true-negative & 200 \\
\hline Total & 21 & 196 & 217 \\
\hline
\end{tabular}

Seventeen lymph node stations measured $1 \mathrm{~cm}$ or greater in short axis at CT scan. This yielded TP in 10, FP in 7, FN in 11, and TN in 189 (Table 2). The sensitivity, specificity, accuracy, positive predictive value, and negative predictive value of PET/CT scan were $81 \%, 92 \%, 91 \%, 53 \%$, and $98 \%$, respectively, whereas those of CT scan were $48 \%, 96 \%, 91 \%, 59 \%$, and $94 \%$ (Table 3). PET/CT scan showed significantly higher sensitivity than CT scan $(\mathrm{p}=0.024)$.

Table 3. Diagnostic results of PET/CT scan and CT scan

\begin{tabular}{lccc}
\hline Variable & PET/CT & CT & Difference \\
\hline Sensitivity & 0.81 & 0.48 & $\mathrm{P}=.024$ \\
Specificity & 0.92 & 0.96 & 0.08 \\
Accuracy & 0.91 & 0.91 & 0.86 \\
Positive predictive value & 0.53 & 0.59 & 0.93 \\
Negative predictive value & 0.98 & 0.94 & 0.15 \\
\hline
\end{tabular}


Table 4 shows a comparison of lymph node staging by PET/CT scan and CT scan with that by pathologic lymph node staging. The accuracy of lymph node staging was $83 \%$ at PET/CT scan and $69 \%$ at CT scan $(p=0.124)$. However, the identification of N2 disease was 8 of $8(100 \%)$ at PET/CT scan, but 3 of $8(37.5 \%)$ at CT scan. We could diagnose the N2 disease at PET/CT scan more accurately than at CT scan $(\mathrm{p}=0.0031)$.
A representative case with a both PET and CT true-positive result is shown in Figure 1. FDG was accumulated in pretracheal lymph node (\#3) at $\mathrm{PET} / \mathrm{CT}$ scan. The size and maximum SUV values were $11.0 \mathrm{~mm}$ and 6.2 . The lymph node was surgically dissected and diagnosed as metastasis.

There were 4 false-negative lymph nodes in 3 cases (Table 5). All of these measured less than 7 $\mathrm{mm}$ in diameter and showed small metastatic areas.

Table 4. Comparison of $\mathrm{N}$ staging with PET/CT scan and CT scan

\begin{tabular}{|c|c|c|c|c|c|c|c|c|c|c|}
\hline & \multicolumn{5}{|c|}{ PET/CT diagnosis } & \multicolumn{5}{|c|}{ CT diagnosis } \\
\hline & N0 & N1 & N2 & N3 & Total & N0 & N1 & N2 & N3 & Total \\
\hline \multicolumn{11}{|c|}{ Pathologic N Stage } \\
\hline No & 26 & 2 & 4 & 0 & 32 & 25 & 5 & 2 & 0 & 32 \\
\hline $\mathrm{N} 1$ & 1 & 1 & 0 & 0 & 2 & 1 & 1 & 0 & 0 & 2 \\
\hline $\mathrm{N} 2$ & 0 & 0 & 8 & 0 & 8 & 4 & 1 & 3 & 0 & 8 \\
\hline N3 & 0 & 0 & 0 & 0 & 0 & 0 & 0 & 0 & 0 & 0 \\
\hline Total & 27 & 3 & 12 & 0 & 42 & 30 & 7 & 5 & 0 & 42 \\
\hline
\end{tabular}
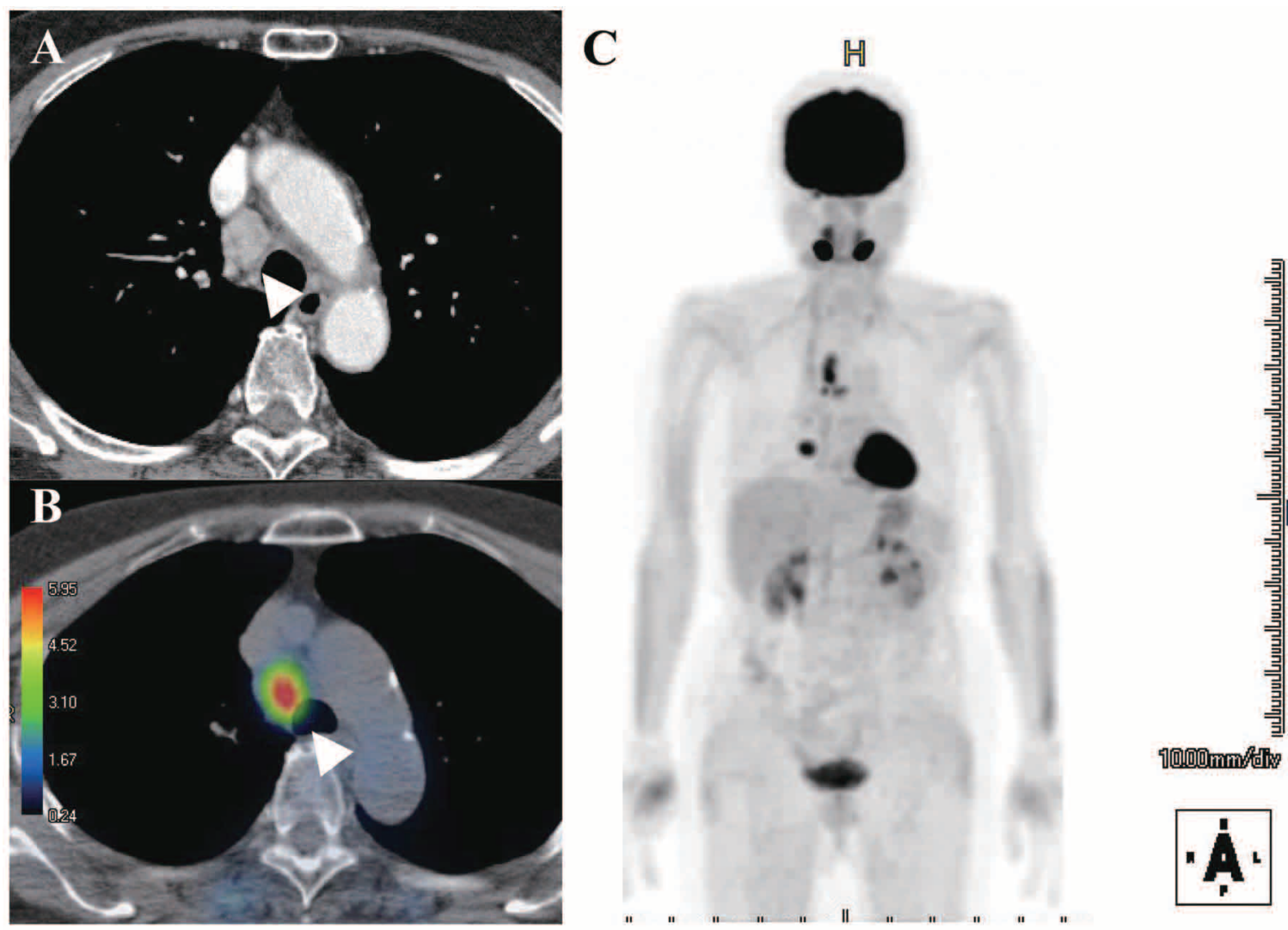

Figure 1. Representative case of true-positive FDG-PET/CT scan and chest CT scan A : Chest CT scan demonstrated the significant swelling of pretracheal lymph node (\#3).

B : PET/CT scan demonstrated the accumulation of FDG (arrow) in the pretoracheal lymph node (\#3).

$\mathrm{C}$ : The view of maximum intensity projection (MIP) of PET/CT scan demonstrated the accumulation of FDG in primary tumor and mediastinal lymph nodes. 
Table 5. False negative PET/CT findings

\begin{tabular}{ccccc}
\hline NO. & Age/sex & Histologic type & Station and size & $\begin{array}{c}\text { The percentage of metastatic area in } \\
\text { largest section of the lymph node }\end{array}$ \\
\hline 1 & $69 /$ male & Ad & $\# 3 / 5.4 \mathrm{~mm}$ & $1 \%$ \\
2 & $31 /$ female & $\mathrm{Sq}$ & $\# 5 / 4.8 \mathrm{~mm}$ & $20 \%$ \\
& & $\# 10 / 5.0 \mathrm{~mm}$ & $60 \%$ \\
3 & & $\mathrm{Sq}$ & $\# 10 / 7.0 \mathrm{~mm}$ & $<5 \%$ \\
\hline
\end{tabular}

$\mathrm{Ad}=$ Adenocarcinoma, $\mathrm{Sq}=$ Squamous cell carcinoma

$\# 3=$ Pretracheal lymph nodes, $\# 5=$ Subaortic lymph nodes, $\# 10=$ Hilar lymph nodes

A representative case with a PET false-negative result is shown in Figure 2. A 75-year-old male (patient 3 in Table 5) with squamous cell carcinoma received preoperative PET/CT scan. FDG was not accumulated in mediastinal and hilar lymph nodes and clinical $\mathrm{N}$-stage was diagnosed as NO. He underwent surgical resection of the primary tumor and lymph node dissection. Histopathologically, right hilar lymph node station (\#10) showed involvement of tumor cells and pathological N-stage was diagnosed as N1. The size of the metastatic lymph node was $7 \mathrm{~mm}$ in diameter and the metastatic foci occupied less than $5 \%$ of the total in the largest section of the lymph node.

There were 15 false-positive lymph nodes in 8 cases. They were 8 mediastinal and 7 hilar lymph nodes. Median size of the lymph nodes was between 4.6 and $11.0 \mathrm{~mm}$ (mean \pm SD, $7.5 \pm 1.9 \mathrm{~mm}$ ). Maximum SUV was between 1.9 and 5.2 (mean \pm SD, $3.4 \pm 1.6)$. Their histological characteristics were lymphoid follicular hyperplasia with anthracosis, calcification, and anthrasilicosis (Table 6). All 8
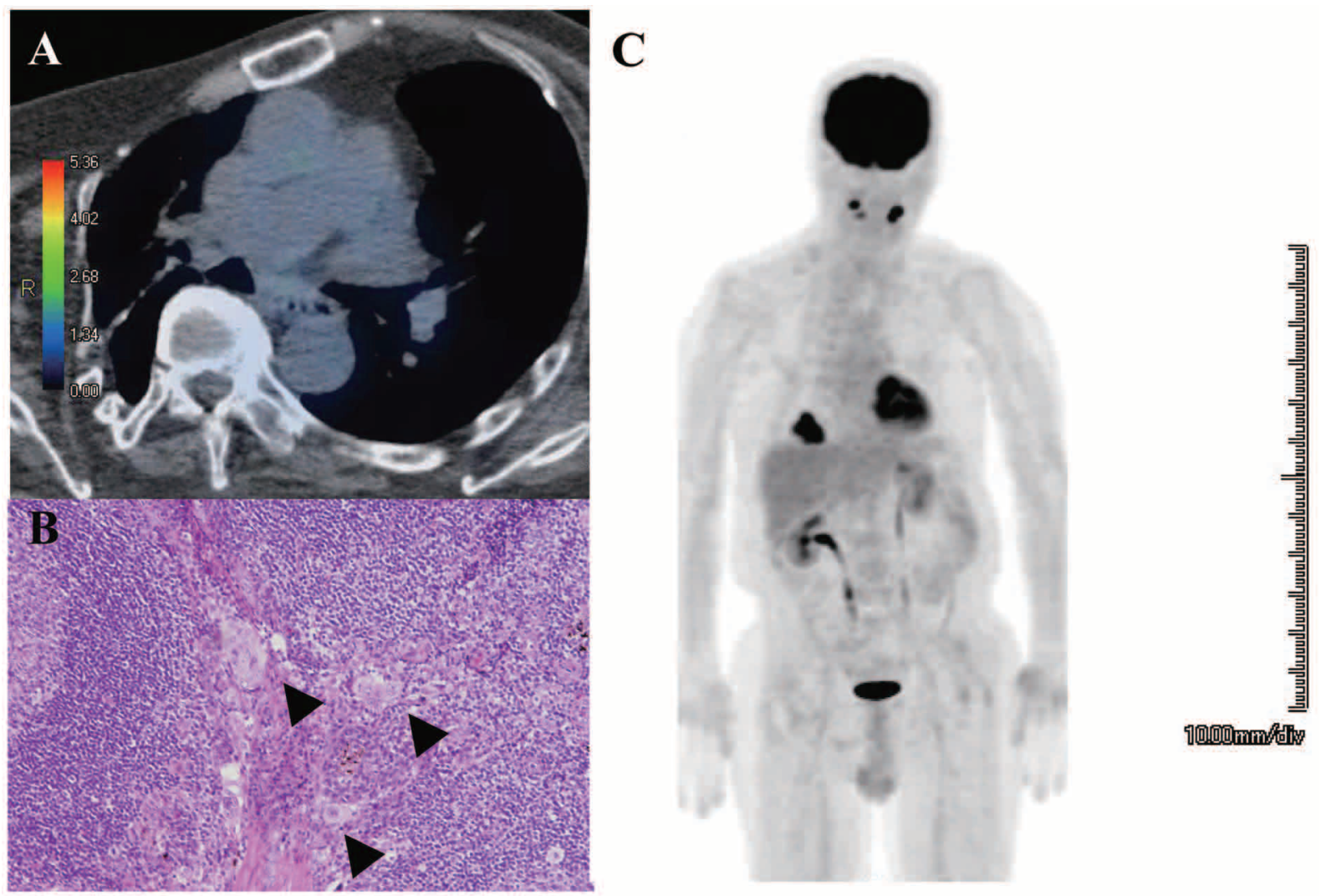

Figure 2. Representative case of false-negative FDG-PET/CT scan

A : FDG was not accumulated in the hilar lymph node (\#10) at PET/CT scan.

B : Microscopic appearance of lymph nodes (hematoxylin and eosin staining, original magnification $\times 100$ ). A few tumor cells (arrow) were recognized in the largest section of the lymph node.

$\mathrm{C}$ : The view of maximum intensity projection (MIP) of PET/CT scan demonstrated no accumulation of FDG in the mediastinal and hilar lymph nodes. 
Table 6. False positive PET/CT findings

\begin{tabular}{|c|c|c|c|c|c|c|}
\hline No. & Age/sex & Station and size & Maximum SUV & Histologial findings of lymph node & Past history & B.I. \\
\hline 1 & $56 /$ male & $\# 11 / 11.0 \mathrm{~mm}$ & 5.2 & anthracosis & & 630 \\
\hline \multirow[t]{2}{*}{2} & $65 /$ male & $\# 3 / 6.7 \mathrm{~mm}$ & 3.1 & anthrasilicosis & Silicosis & 4600 \\
\hline & & $\# 11 / 6.8 \mathrm{~mm}$ & 3.6 & anthrasilicosis & & \\
\hline 3 & $69 /$ male & $\# 11 / 5.0 \mathrm{~mm}$ & 3.1 & anthracosis & & 800 \\
\hline 4 & $69 /$ male & $\# 11 / 9.7 \mathrm{~mm}$ & 3.1 & anthracosis & & 1820 \\
\hline \multirow[t]{2}{*}{5} & $67 /$ male & $\# 3 / 9.5 \mathrm{~mm}$ & 2.8 & anthracosis & & 640 \\
\hline & & $\# 7 / 6.2 \mathrm{~mm}$ & 3.2 & anthracosis & & \\
\hline \multirow[t]{2}{*}{6} & $64 /$ male & $\# 5 / 6.0 \mathrm{~mm}$ & 3.5 & anthrasilicosis & Silicosis & 740 \\
\hline & & $\# 11 / 6.8 \mathrm{~mm}$ & 8.6 & anthrasilicosis & & \\
\hline \multirow[t]{2}{*}{7} & 71/female & $\# 5 / 7.3 \mathrm{~mm}$ & 2.6 & anthrasilicosis & & 300 \\
\hline & & $\# 6 / 6.7 \mathrm{~mm}$ & 2.6 & anthrasilicosis & & \\
\hline \multirow[t]{4}{*}{8} & $69 /$ male & $\# 3 / 7.2 \mathrm{~mm}$ & 1.9 & anthracosis, calcification & Tuberculosis & 800 \\
\hline & & $\# 7 / 4.6 \mathrm{~mm}$ & 2.4 & anthracosis, calcification & & \\
\hline & & $\# 10 / 8.6 \mathrm{~mm}$ & 2.3 & anthracosis, calcification & & \\
\hline & & $\# 11 / 9.7 \mathrm{~mm}$ & 2.4 & anthracosis, calcification & & \\
\hline
\end{tabular}

$\# 3=$ Pretracheal lymph nodes, $\# 5=$ Subaortic lymph nodes, $\# 6=$ Paraaortic lymph nodes \#7=Subcarinal lymph nodes, \#10=Hilar lymph nodes, \#11= Interlobar lymph nodes B.I. $=$ Brinkman Index

cases with false-positive results on FDG-PET/CT scan had smoking history (B.I. $=300-4600), 2$ cases (25.0\%) had silicosis, and one case (12.5\%) had had tuberculosis in the past. A representative case with a PET false-positive result is shown in Figure 3.
A 69-year-old male (patient 8 in Table 6) with adenocarcinoma received preoperative PET/CT scan. FDG was accumulated in mediastinal and hilar lymph node stations (\#3, \#7, \#10, \#11) clinical Nstage was diagnosed as N2. Sizes and maximum
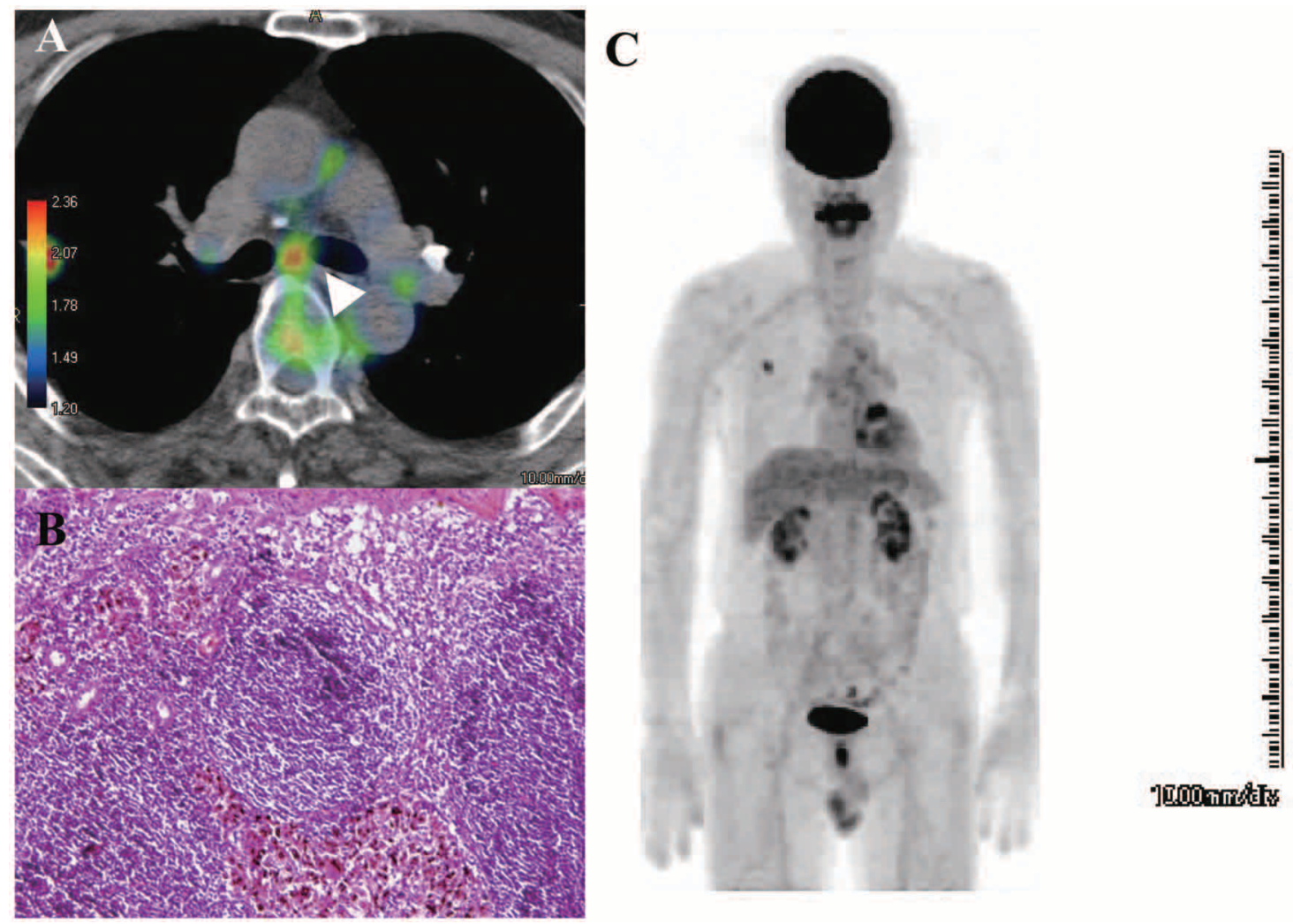

Figure 3. Representative case of false-positive FDG-PET/CT scan

A : PET/CT scan demonstrated accumulation of FDG (arrow) in the subcarinal lymph node (\#7).

B : Microscopic appearance of lymph nodes (hematoxylin and eosin staining, original magnification $\times 100$ ). Follicular lymphoid hyperplasia with anthrasilicosis and no tumor cells in the lymph nodes were revealed.

$\mathrm{C}$ : The view of maximum intensity projection (MIP) of PET/CT scan demonstrated the accumulation of FDG in primary tumor and mediastinal and hilar lymph nodes. 
SUV values of the lymph nodes (\#7) were $4.6 \mathrm{~mm}$ in diameter and 2.4. But because the degree of accumulation of FDG in each lymph node station was almost same compared with background activity in surrounding normal tissue in the view of maximum intensity projection (MIP), the possibility of the N0 disease was suggested. He underwent surgical resection of the primary tumor and lymph node dissection. Histopathologically, dissected lymph nodes showed follicular lymphoid hyperplasia with anthrasilicosis and no involvement of tumor cells ; pathological N-stage was diagnosed as N0.

Figure 4 shows the relationship between metastatic states and the size of PET-positive lymph nodes. If the cut-off value of the size of lymph nodes was set at $1 \mathrm{~cm}$ in diameter, 9 (90\%) of $10 \mathrm{lymph}$ node stations with PET-positive lymph nodes of more than $1 \mathrm{~cm}$ showed metastasis. However, metastasis was shown in only 8 (36\%) of 22 lymph node stations with PET-positive lymph nodes of less than $1 \mathrm{~cm}$. The positive predictive value of lymph nodes measuring more than $1 \mathrm{~cm}$ in diameter was significantly higher than that of lymph nodes measuring less than $1 \mathrm{~cm}$ in diameter ( 0.90 vs. $0.36, \mathrm{p}=0.015)$.

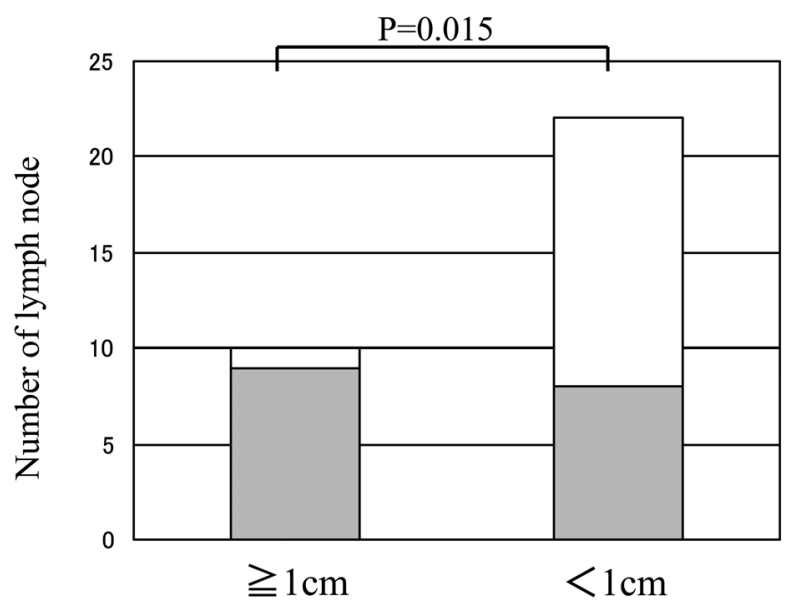

Size of PET-positive lymph nodes

Figure 4. The relationships between metastatic state and the size of lymph nodes at

FDG-PET/CT scan. The positive predictive value of lymph nodes of more than $1 \mathrm{~cm}$ in diameter was higher than that of those of less than $1 \mathrm{~cm}$ in diameter ( 0.90 vs. $0.36, \mathrm{p}=0.015)$.

True-positive cases $\square$ False-positive cases

\section{DISCUSSION}

In this study, the sensitivity of PET/CT scan was significantly higher than that of only CT scan $(81 \%$ vs. $48 \%, \mathrm{p}=0.024)$. On the other hand, the specificity and accuracy of both modalities were similar. Concerning lymph node staging, there were no significant differences between FDG-PET/CT scan and CT scan. However, concerning the identification of N2 disease, which has sometimes been a clinical problem of therapeutic strategy, FDG-PET/CT scan was significantly more accurate than CT scan.

Many authors have reported that FDG-PET/CT scan was effective with regard to lymph node staging in patients with lung cancer and the sensitivity and specificity were almost $80-90 \%$ and were generally superior to those of CT scan (1-6, 9). Our data was similar to previous reports and the sensitivity of PET/CT scan was significantly superior to CT scan although there was no difference between FDG-PET/CT scan and CT scan with regard to the specificity. It is recommended that FDG-PET/ CT scan should be performed as a non-invasive diagnostic tool in ACCP guidelines (5) and ESTS guidelines (10). In view of our data, it is thought that the FDG-PET/CT scan is currently the most reliable non-invasive diagnostic tool with regard to the presence of lymph node metastasis and should be performed preoperatively as a routine examination.

In this study, the PPV of FDG-PET/CT scan was $53 \%$. There were 8 cases with 15 false-positive lymph node stations at FDG-PET/CT scan. All falsepositive lymph nodes showed lymphoid follicular hyperplasia with anthracosis, anthrasilicosis, and calcification histologically. In addition, all patients with false-positive lymph node stations were smokers.

Several previous studies have reported that the causes of false-positive accumulation of FDG in lymph nodes may be the infiltration of activated macrophages and inflammatory cells in conditions such as active tuberculosis and granuloma (11-13). Lymphoid follicular hyperplasia is a type of chronic lymphadenopathy and Chung JH et al. (14) reported that the overexpression of glucose transporter 1 (Glut 1), which is known to be related to FDG uptake, was recognized in lymph nodes with the finding of lymphoid follicular hyperplasia. Therefore, some authors mentioned that the presence of lymphoid follicular hyperplasia was possibly related to false-positive results on FDG-PET/CT scan (14, 15). Chung JH et al. (14) also mentioned that patients with heavy smoking, chronic airway disease, or tuberculosis might have mediastinal lymphoid follicular hyperplasia. This would be compatible with our findings because our cases with false-positive 
results on FDG-PET/CT scan had a smoking history and concomitant lung diseases such as silicosis and previous pulmonary tuberculosis. In our cases, if false-positive cases with those factor were excluded, The specificity, accuracy, positive predictive value of PET/CT scan were $98 \%, 97 \%$ and $85 \%$ and they were more higher. Furthermore the accuracy of PET/CT scan was also significantly higher than that of CT scan $(p=0.023)$. So we have to judge the findings of PET/CT scan in consideration of clinical background.

Our study suggested that the PPV of lymph nodes measuring more than $1 \mathrm{~cm}$ in diameter was significantly higher than that of those measuring less than $1 \mathrm{~cm}$ in diameter. Therefore, if there is accumulation of FDG in lymph nodes of more than $1 \mathrm{~cm}$, we will be able to diagnose metastasis at a high rate. However, if there is accumulation of FDG in lymph nodes measuring less than $1 \mathrm{~cm}$, invasive modalities such as mediastinoscopy, endobronchial ultrasonography transbronchial needle aspiration (EBUSTBNA), esophageal endoscopic ultrasound-guided fine needle aspiration (EUS-FNA), and video-assisted thoracic surgery (VATS) should be considered to decide the indication for curative operation (16).

The negative predictive value (NPV) of FDGPET/CT scan was high. However, it is difficult to diagnose as false-negative for lymph nodes in which FDG is not found to have accumulated at PET/CT scan. In our false-negative cases, the sizes of these lymph nodes were small, less than $7 \mathrm{~mm}$ in diameter, or a small metastatic area in the lymph node was histologically identified. As they were incidental lymph node metastases, we could not preoperatively diagnose the presence of metastasis. Therefore, there are limitations of the diagnostic capacity of FDG-PET/CT scan especially in cases with micrometastasis and small lymph nodes. If FDG was not found to have accumulated in the lymph nodes at PET/CT scan, we should diagnose as $\mathrm{cN} 0$ and make the decision on therapeutic principle. If incidental lymph node metastases of $\mathrm{N} 1$ or $\mathrm{N} 2$ station are found on final pathology examination of the resected specimen, we should treat by using surgical resection followed by adjuvant chemotherapy (17).

In conclusion, FDG-PET/CT scan is more accurate than $\mathrm{CT}$ scan concerning the diagnosis of lymph node metastasis and staging. However, we have to judge the findings in consideration of clinical background because false-positive results on
FDG-PET/CT scan are often recognized. We should recognize the limitations of the diagnostic capacity of FDG-PET/CT and select an appropriate therapy. When there is accumulation of FDG in lymph nodes of more than $1 \mathrm{~cm}$ in diameter, we should judge as true-positive and decide the appropriate therapeutic strategy. When there is accumulation of FDG in the lymph nodes of less than $1 \mathrm{~cm}$ in diameter, we have to make their histological diagnoses using EBUS-TBNA, EUS-FNA, mediastinoscopy and VATS if we are obliged to change our therapeutic strategy by the presence of metastasis. When there is no accumulation of FDG in lymph nodes, we should judge as true-negative and consider the surgical approach.

\section{REFERENCES}

1. Shim SS, Lee KS, Kim BT, Chung MJ, Lee EJ, Han J, Choi JY, Kwon OJ, Shim YS, Kim S : Non-small Cell Lung cancer : Prospective Comparison of Integrated FDG PET/CT and CT alone for Preoperative Staging. Radiology 236 : 1011-1019, 2005

2. Cerfolio RJ, Bryant AS, Ohja B, Bartolucci AA : The maximum standardized uptake values on positron emission tomography of a non-small cell lung cancer predict stage, recurrence, and survival. J Thorac Cardiovasc Surg 130 : 151-9, 2005

3. Gould MK, Kuschner WG, Rydzak CE, Maclean CC, Demas AN, Shigemitsu H, Chan JK, Owens DK : Test Performance of Positron Emission Tomography and Computed Tomography for Mediastinal Staging in Patients with Non-SmallCell Lung Cancer. Ann Intern Med 139 : 879892, 2003

4. van Tinteren $\mathrm{H}$, Hoekstra OS, Smit EF, van den Bergh JH, Schreurs AJ, Stallaert RA, van Velthoven PC, Comans EF, Diepenhorst FW, Verboom P, van Mourik JC, Postmus PE, Boers $\mathrm{M}$, Teule GJ : Effectiveness of positron emission tomography in the preoperative assessment of patients with suspected non-small-cell lung cancer : the PLUS multicentre randomized trial. Lancet 359 : 1388-92, 2002

5. Birm Ö, Kappetein AP, Stijnen T, Bogers AJJC : Meta-Analysis of Positron Emission Tomographic and Computed Tomographic Imaging in Detecting Mediastinal Lymph Node Metastases in Nonsmall Cell Lung Cancer. Ann 
Thorac Surg 79 : 375-81, 2005

6. Silvestri GA, Gould MK, Margolis ML, Tanoue LT, McCrory D, Toloza E, Detterbeck F. Noninvasive Staging of Non-small Cell Lung Cancer. Chest $132: 178-201,2007$

7. Naruke T, Suemasu K, Ishikawa S. Lymph node mapping and curability at various levels of metastasis in resected lung cancer. J Thorac Cardiovasc Surg 76: 832-9, 1978

8. McLoud TC, Bourgouin PM, Greenberg RW, Kosiuk JP, Templeton PA, Shepard JA, Moore EH, Wain JC, Mathisen DJ, Grillo HC : Bronchogenic carcinoma : analysis of staging in the mediastinum with CT by correlative lymph node mapping and sampling. Radiology 182 : 319-323, 1992

9. Glazer GM, Gross BH, Aisen AM, Quint LE, Francis IR, Orringer MB : Imaging of the pulmonary hilum : a prospective comparative study in patients with lung cancer. AJR Am J Roentgenol 145 : 245-248, 1985

10. Leyn PD, Lardinois D, van Schil PE, RamiPorta R, Passlick B, Zielinski M, Waller DA, Lerut T, Weder W : ESTS guidelines for preoperative lymph node staging for non-small cell lung cancer. Eur J Cardiothorac Surg 32 : 18,2007

11. Gupta NC, Graeber M, Rogers JF, Bishop HA : Comparative efficacy positron emission tomography with FDG and computed tomographic scanning in preoperative staging of non-small cell lung cancer. Ann Surg 229 : 286-291, 1999

12. Farrell MA, McAdams HP, Herndon JE, Patz EF : Non-small cell lung cancer : FDG PET for nodal staging in patients with stage I disease. Radiology 215 : 886-90, 2000

13. Roberts PF, Follette DM, von Haag D, Park JA, Valk PE, Pounds TR, Hopkins DM : Factors associated with false-positive staging of lung cancer by positron emission tomography. Ann Thorac Surg $70: 1154-9$ (discussion 9-60), 2000

14. Chung JH, Cho KJ, Lee SS, Baek HJ, Park JH, Cheon GJ, Choi CW, Lim SM : Overexpression of Glut 1 in Lymphoid Follicles Correlates with False-Positive ${ }^{18} \mathrm{~F}$-FDG PET Results in Lung Cancer Staging. J Nucl Med 45 : 999-1003, 2004

15. Takamochi K, Yoshida J, Murakami K, Niho S, Ishii G, Nishimura M, Nishiwaki Y, Suzuki K, Nagai K : Pitfalls in lymph node staging with positron emission tomography in non-small cell lung cancer patients. Lung Cancer 47 : 235242, 2005

16. Detterbeck FC, Jantz M, Wallace M, Vansteenkirste J, Silvestri GA : Invasive Mediastinal Staging of Lung Cancer. Chest 132 : 202-220, 2007

17. Van Meerbeeck JP, Surmont Veerle FM : Stage IIIA-N2 NSCLC : A review of its treatment approaches and future developments. Lung Cancer 65 : 257-267, 2009 